\title{
Morphology of the tracheobronchial tree of the Ganges river dolphin (Platanista gangetica)
}

\author{
By \\ Masahiko Y. KIDA \\ Department of Anatomy, Sapporo Medical College, Sapporo, 060 Japan \\ - Received for Publication, May 24, 1990-
}

\begin{abstract}
Key words: Tracheobronchial tree, Tracheal bronchus, Ganges river dolphin, Comparative anatomy
Summary: To contribute to the phylogenetic evaluation of the river dolphin, the morphology of the tracheobronchial tree of the Ganges river dolphin was analyzed according to Nakakuki's fundamental bronchial tree model of mammals. In the right lung, the bronchial tree consists of the tracheal bronchus, five lateral series bronchi, three dorsal series bronchi and one ventral series bronchus. In the left lung, it consists of five lateral series bronchi, four dorsal series bronchi and one ventral series bronchus. In the Ganges river dolphin, the tracheal bronchus corresponds to type III of Nakakuki's nomenclature. This characteristic is of especial interest in light of the fact that the Ganges river dolphin has a forestomach as do Artiodactyla.
\end{abstract}

The biological characteristics of fresh-water dolphins have been widely examined ever since the first taxonomical study was reported by Gray (1863). In the world today, four living genera of these dolphins are recognized after the first report on Lipotes by Miller (1918). All interpretations of the phylogenetic relation among the four genera depend on differences caused by regarding what kinds of characteristics as being more important (Kaiya, 1982). Therefore, the visceral organs of river dolphins have been examined and reported in addition to their skeletal system. However, very little is known about the details of the tracheobronchial trees of river dolphins, and they have never been discussed with reference to Nakakuki's theory which phylogenetically interprets ramification patterns of mammalian bronchi (Nakakuki, 1980). Here the author reports on the morphology of the tracheobronchial tree of the Ganges river dolphin on the basis of Nakakuki's theory in order to contribute to the phylogenetic evaluation of the dolphin.

\section{Materials and Methods}

A pair of lungs of the Ganges river dolphin was examined in this study. This dolphin was collected by the Cetacean Research Expedition, the University of Tokyo, at Bhabakhali, Bangladesh in 1970. It was "emale, and its body length and body weight were 76 im and $8.4 \mathrm{~kg}$, respectively.

The lungs were preserved at Sapporo Medical
College in $10 \%$ formalin solution. They were carefully dissected and their parenchyma was manually removed to examine the tracheobronchial tree and the ramifications of the blood vessels. The findings of these organs were recorded by means of sketches and photographs.

\section{Observations}

The right and left lungs of this animal are $15.1 \mathrm{~cm}$ and $14.0 \mathrm{~cm}$ in maximum length, and $7.3 \mathrm{~cm}$ and $8.1 \mathrm{~cm}$ in maximum width, respectively (Plt. 1). The trachea separates into three bronchi, namely the tracheal bronchus and the right and left main bronchi (Plt. 2). The bronchial tree consists of a long trunk and several branches in either side distal to the tracheal bifurcation (Figs. $1 \& 2$ ). This is different from the morphology of the human bronchial tree. The Ganges river dolphin has more segmental bronchi than humans. For convenience of explanation and for objectivity, the present author describes findings using the terms "trunk and branch" instead of "lobar or segmental bronchus" in this study.

In the right lung, the tracheal bronchus ramifying directly from the trachea distributes to the cranial portion (apex) of the lung. The branches ramify metamerically from the right trunk, although it is certainly difficult to differentiate distinctly which bronchus is the trunk in its distal portion (Fig. $1 \&$ Plt. 2). The ramifying positions of branches from the trunk make three independent longitudinal series, namely the lateral, 


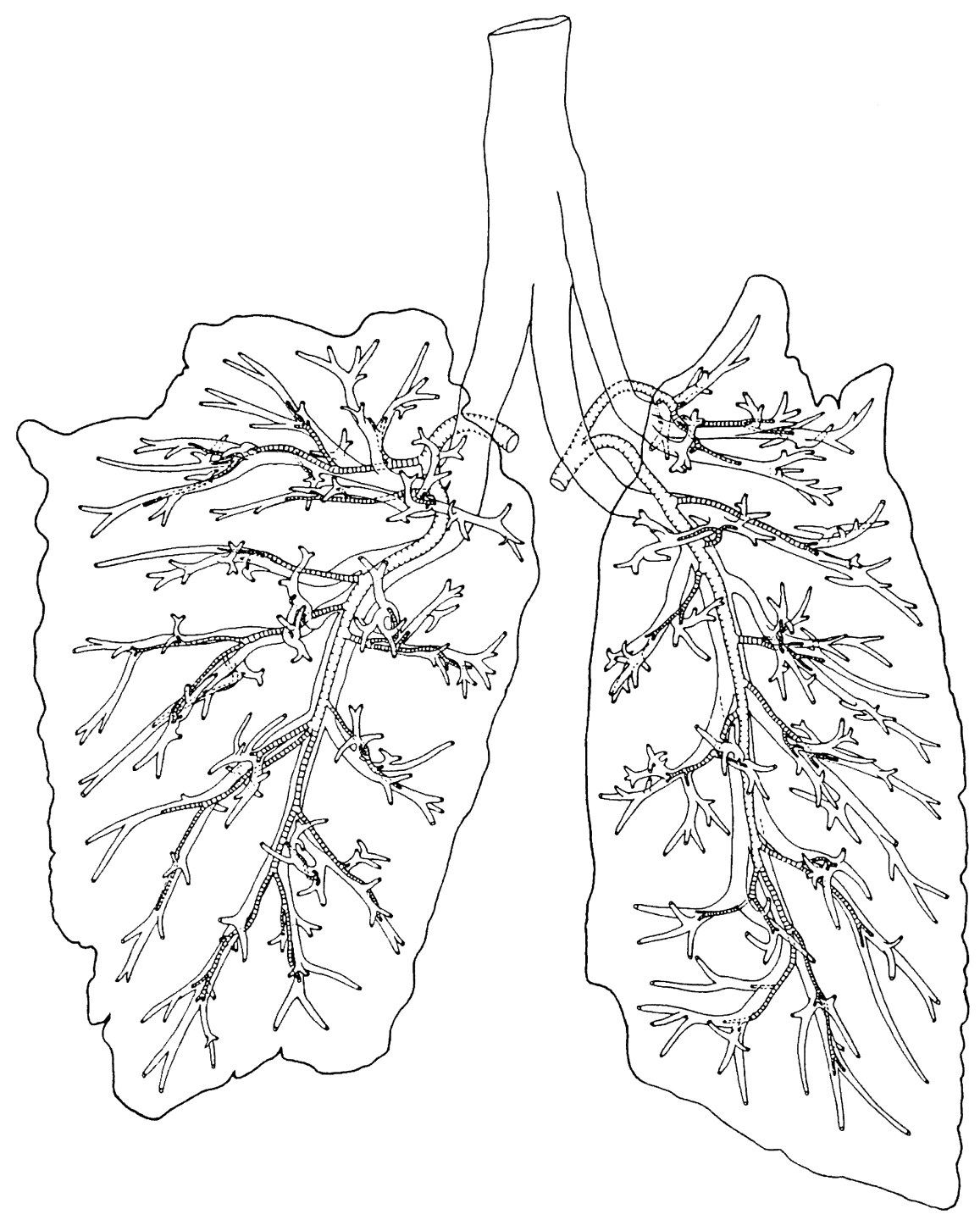

Fig. 1. Morphology of the tracheobronchial tree from the dorsal aspect with distribution of the pulmonary arterial system.

dorsal and ventral series. With the exception of the tracheal bronchus, the lateral, dorsal and ventral series consist of five, three and one primary branches, respectively. The line tracing distalwards the ramifying positions of the dorsal series branches shows slight clockwise spiral in the cranial view. It is easy to recognize this spiraled course of the line as contrasted with the course of the lateral series by means of plotting the ramifying positions of branches on the wall plan of the longitudinally opened trunk (Fig. 3). These two lines tracing the ramifying positions of the dorsal and lateral series gradually separate from each other towards the tip of the trunk. The ramifying position of the tracheal bronchus is not located exactly on the lateral side of the trachea but deviates slightly dorsally. The branches of the dorsal and lateral series develop well and occupy almost all parts of the lungs.

Every primary branch of the lateral series has three secondary branches (Fig. 1). It first leaves a secondary branch dorsally and then bifurcates to become cranial and caudal secondary branches. With regard to the dorsal series, a primary branch separates into two secondary branches, namely the lateral and medial branches. Concerning the right first and the left first and second branches of the dorsal series, the medial secondary branches further bifurcate immediately after the secondary ramification, however these branches on first glance appear to separate into three secondary branches.

In the left lung, there is no tracheal bronchus and the number of primary branches differs from that in the right lung. The lateral, dorsal and ventral series con- 


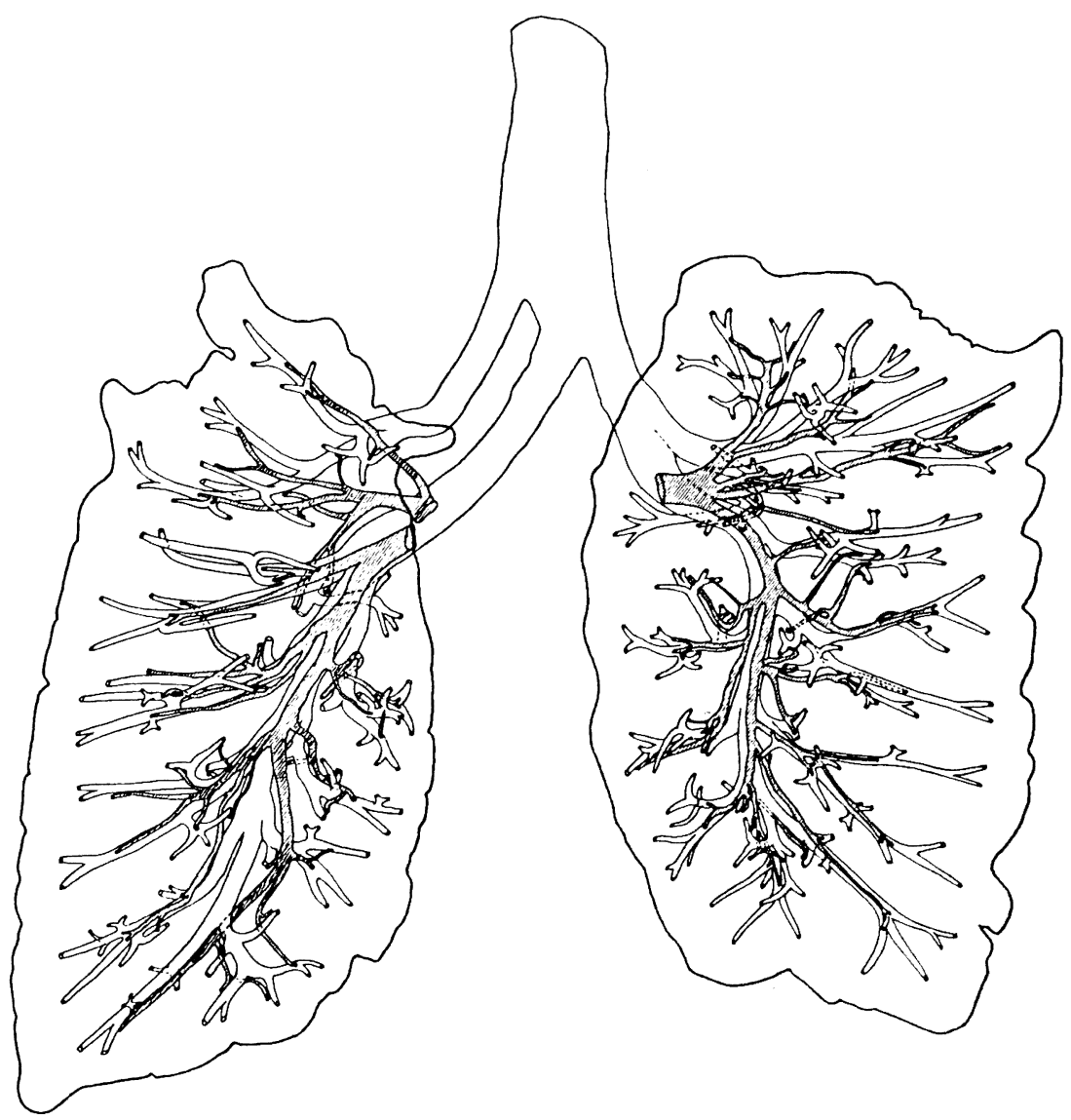

Fig. 2. Morphology of the tracheobronchial tree from the ventral aspect with distribution of the pulmonary venous system.

sist of five, four and one primary branches, respectively. Apart from the above, the other characteristics clearly different from the right is that the line tracing the ramifying positions of the dorsal series bronchi slightly spirals in a counterclockwise direction in contrast with that of the right side.

As far as pulmonary vessels are concerned, the arterial and the venous systems principally run along the dorsal and ventral sides of the bronchial tree, respectively (Figs. $1 \& 2$ ). The pulmonary arteries run more closely along the bronchi than do the pulmonary veins. The right pulmonary artery has two main branches. The cranial one winds from ventral dorsalwards over the tracheal bronchus and the caudal one over the proximal part of the right trunk prior to ramification of the first branch. The left pulmonary artery winds over the left trunk as the same manner of the caudal branch of the right pulmonary artery.

With regard to the courses of the veins draining the lobules distributed by the caudalmost lateral branch or the distal portion of the trunk, they do not agree with the previously mentioned principle. In case of the caudal lobules of the right lung distributed by both the caudalmost lateral branch and the distal portion of the trunk, the veins run along the dorsal side of the bronchi and arteries. In case of the caudal lobules of the left lung distributed by the caudalmost lateral branch the two different courses of the veins draining these lobules are observable, namely they run along the ventral and dorsal sides of both the bronchus and the artery.

\section{Discussion}

The current nomenclature of the tracheobronchial system cannot be appropriately applied to mammals except for humans because it is devised clinically based on human lungs (Jackson \& Huber, 1943; Yamashita, 1978). Namely, the nomenclature is not systematic from the view point of comparative anatomy as Nakakuki (1980) has already pointed out. He has examined the ramifying positions of the primary branches from the trunk in many kinds of mammals and has given a phylogenetic interpretation and a new nomenclature for the tracheobronchial system (Nakakuki, 1980 \& 1988). His nomenclature is employed in this study because his 

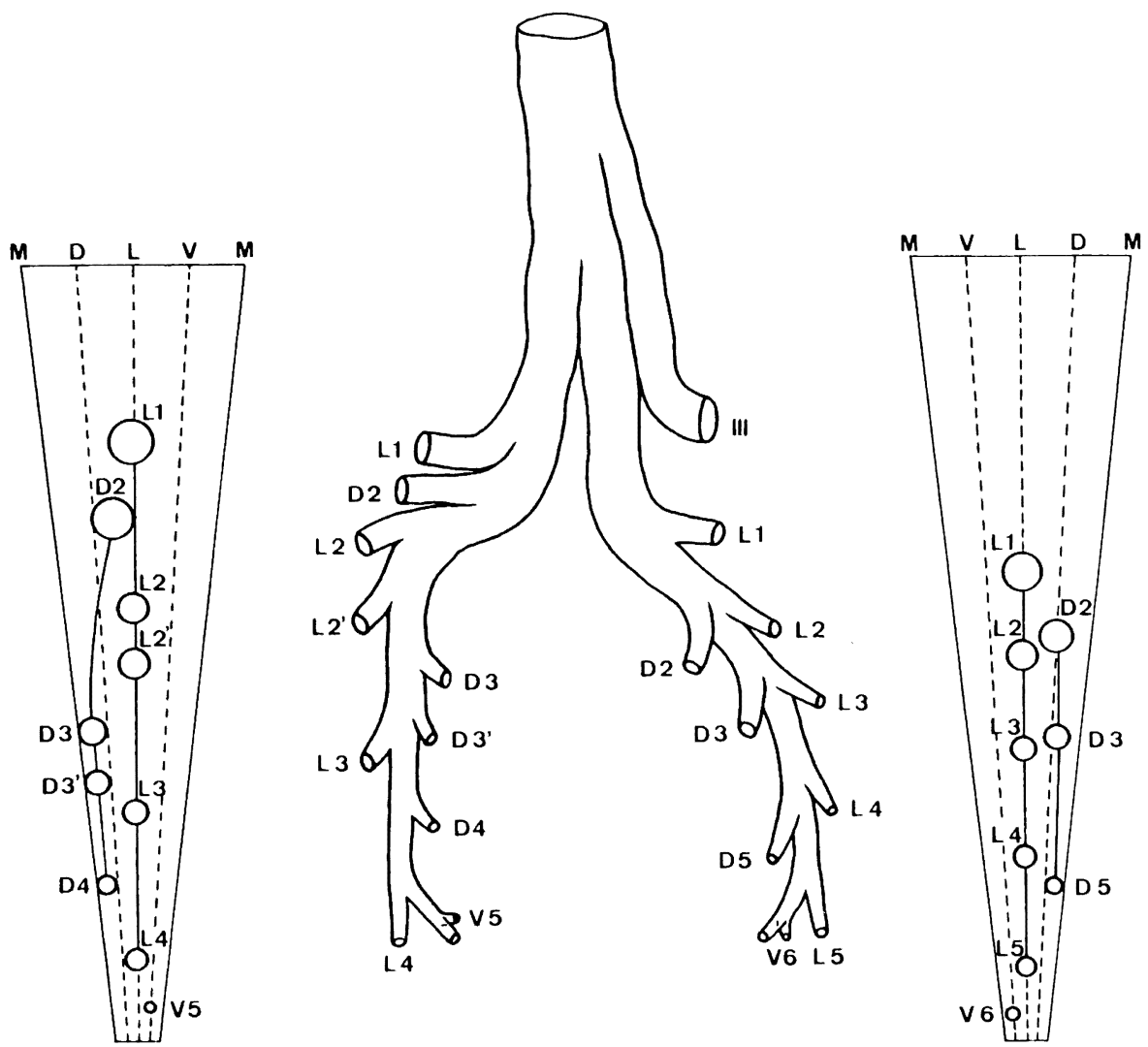

Fig. 3. Diagram of the tracheobronchial tree in the dorsal aspect to show the separating positions of branches from the trunk. They are displayed on the wall surface of the longitudinally opened trunk. III: The highest ramification type of tracheal bronchus ramification, L: the lateral series, $\mathrm{D}$ : the dorsal series and $\mathrm{V}$ : the ventral series.

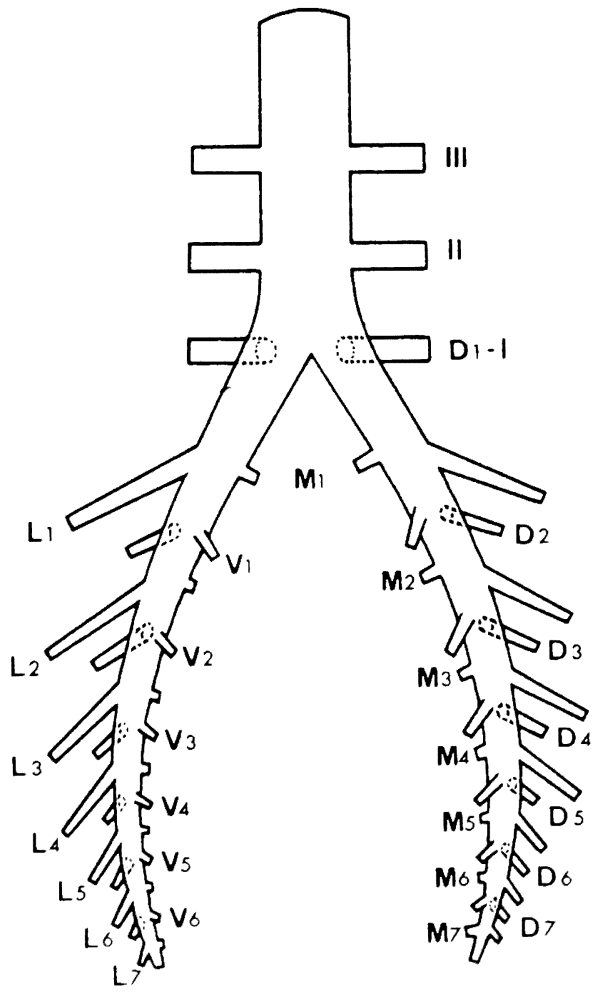

theory seems to be the most overall acceptable and reliable for mammals in general.

The morphology of the tracheobronchial tree of the Ganges river dolphin can be interpreted relatively well as those of mammals on the basis of Nakakuki's theory. According to his theory, the trunk consists of the bronchial parts which correspond to the human main bronchus, truncus intermedius, inferior trunk and medial branch of the posterior basal segmental bronchus, using the terms devised by the Japanese Committee on the Nomenclature for Bronchial

Fig. 4. Fundamental tracheobronchial tree model of mammals (Nakakuki, 1980). According to Nakakuki: "There is a definite relation in the ramification of bronchus of mammalian lung, i.e., a series of bronchiole arise longitudinally and metamerically from the dorsal (D), ventral (V), lateral (L) and medial (M) sides of bronchus, constituting the dorsal, ventral, lateral and medial bronchiole systems. On the upper portion from the bifurcated portion of trachea, two pairs of bronchiole of upper lobe (III \& II), which belong probably to the dorsal bronchiole system, arise from the right and left sides of trachea. Further, a pair of upper lobe bronchiole (I), which belong to the dorsal bronchiole system, arise near the bifurcated portion of trachea". 

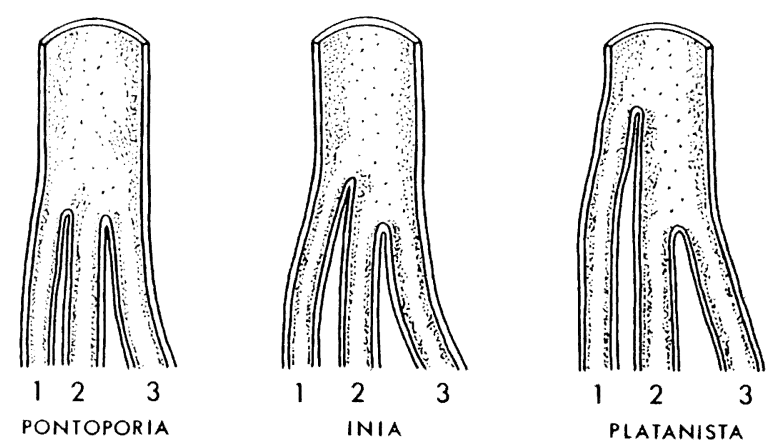

Fig. 5. Three types of tracheal ramification in Platanistidae (modified from Yamasaki et al., 1977). 1: Tracheal bronchus, 2: the right main bronchus and 3: the left main bronchus.

Branching (1952 \& 1962). The bronchial ramifying directions of this dolphin can be considered to consist of the three series out of the four described in Nakakuki's fundamental tracheobronchial tree model (Figs. 3 \& 4). However, intervals between neighboring ramifying positions are not constant among those three series. This irregularity cannot be completely explained by Nakakuki's theory.

The tracheal bronchus is the conspicuous feature of the tracheobronchial tree of the Ganges river dolphin. In the case of this dolphin, this bronchus is regarded as corresponding to type III of Nakakuki's fundamental model of the mammalian tracheobronchial tree because the ramifying level of the tracheal bronchus is the highest among the three kinds of river dolphins, Pontoporia, Inia and Platanista (Fig. 5).

The existence of a tracheal bronchus has been pointed out as being very common in cetaceans. It is also common in the tracheobronchial tree of Artiodactyla (Slijper, 1979). As far as the author could determine from the literature, the tracheal bronchus is certainly observable in Franciscana (Burmeister, 1867; Yamasaki et al. 1977), bottle-nosed dolphin (Fanning \& Harrison, 1974), finless porpoise (Ping, 1926; Qian, 1986), Bouth (Yamasaki et al. 1977) and fin whale (Nakakuki, 1980). It is worth noting that the tracheal bronchi of the Ganges river dolphin and the artiodactyla commonly exist in the right lung and they correspond to type III of Nakakuki's nomenclature. The Amazon river dolphin, Inia, has type II tracheal bronchus. In cattle, type III tracheal bronchus is usually observable, and sometimes accompanied by type II. This is of interest in light of the fact that a forestomach is also observable only in the Ganges river dolphin and the Amazon river dolphin out of the four kinds of river dolphins (Yamasaki \& Kamiya, 1981).

While the tracheal bronchus distributes to the apex of the right lung, corresponding to the right upper lobe, the apex of the left lung is supplied by the first branch of the lateral series. The first lateral branch of the right lung distributes to the lateral middle portion, being equivalent to the middle lobe (In fact, no lobe is recognizable in the lungs of the Ganges river dolphin). Therefore, the apex of the left lung is considered to be equivalent to the middle lobe of the right lung, as has been already pointed out by Nakakuki in many other mammals (Nakakuki, 1980).

As far as the relation between the pulmonary vessels and the bronchial tree is concerned, it is logical to postulate that the pulmonary arterial and venous systems principally run along the dorsal and ventral sides of the bronchial tree, respectively. In spite of existence of the irregular courses of veins draining the caudal lobules of lungs, this postulation can be supported by the coexistence of the two different venous courses running along the ventral and dorsal sides of bronchi observed in the caudal lobules of the left lung. The reason why the coexistence supports the postulation is that it can be regarded as indicating a transitional type for explaining an alternating venous distribution process.

\section{Acknowledgment}

This is a part of the macro-microscopic study of respiratory organs of Ganges river dolphin, the outline of which was presented at the XIII Federative International Congress of Anatomy, Rio de Janeiro, 1989.

Thanks to Dr. F. Yamasaki, former professor of Sapporo Medical College, and Prof. T. Kamiya, College of Medical Technology and Nursing, University of Tsukuba, a pair of lungs of the Ganges river dolphin was available for this study. Professors Yamasaki and Kamiya were members of the Cetacean Research Expedition, the University of Tokyo. The present author is deeply indebted to them. $\mathrm{He}$ is also grateful to Prof. Y. Dodo, Sapporo Medical College, for helpful advice in the course of this study. He expresses his sincere thanks to Prof. S. Nakakuki, Tokyo University of Agriculture and Technology, for his useful advice and to Assoc. Prof. J. P. Barron, St. Marianna University School of Medicine, for grammatical review of the manuscript.

\section{References}

1) Burmeister, H.: Preliminary observations on the anatomy of Pontoporia blainvillii. Proc. Zool. Soc. London, 484-489, 1867.

2) Fanning, J. C.\& Harrison, R. J.: The structure of the trachea and lungs of the South Australian bottle-nosed dolphin. In: harrison R. ed., Functional anatomy of marine mammals. Vol. 2, 231-252. Acad. Press, London and New York, 1974.

3) Gray, J. E.: On the arrangement of the cetaceans. Proc. Zool. Soc. London, 197-202. (cited from Kaiya, 1982)

4) Jackson, C. L. \& Huber, J. F.: Correlated applied anatomy of the bronchial tree and lungs with a system of nomenclature. Dis. Chest., 9: 319-326, 1943. 
5) Kaiya, Z.: Classification and phylogeny of the superfamily Platanistoidea, with notes on evidence of the monophyly of the cetacea. Sci. Rep. Whale Res. Inst., 34: 93-108, 1982.

5) Miller, G. S. Jr.: A new river-dolphin from China, Smiths. Misc. Coll., 68: 1-12, 1918

7) Nakakuki, S.: Comparative anatomical studies on the mammalian lung. Bull. Faculty of Agricul. Tokyo Univ. Agricul. \& Technol., 21: 1-74, 1980.

8) Nakakuki, S.: Mammalian lung, Primate lung, Human lung -Comparative anatomical approach-. Primate Res., 4: 121-133, 1988. (in Japanese)

9) Ping, C.: On some parts of the visceral anatomy of the porpoise, Neomeris phocoenoides. Anat. Rec., 33: 13-28, 1926.
10) Qian, W.: Study on the anatomy and histology of trachea and lung of Neophocaena asiaeorientalis. Acta Theriol. Sinica, 6: 183-189, 1986

11) Slijper, E. J.: Whales 2nd ed. Hutchinson, London, 1979.

12) Yamasaki, Y.\& Kamiya, T.: The stomach of the Boutu, Inia geoffrensis: Comparison with those of other Platanistids. Sci. Rep. Whales Res. Inst., 33: 69-81, 1981.

13) Yamasaki, F., Takahashi, K. \& Kamiya, T.: Lungs of Franciscana (Pontoporia blainvillei), with special references to their external aspects, weights and bronchial ramifications. Okajimas Folia Anat. Japon., 53: 337-357, 1977.

14) Yamashita, H.: Roentgenologic anatomy of the lung, 1st ed. Igaku-shoin, Tokyo, 1978. 


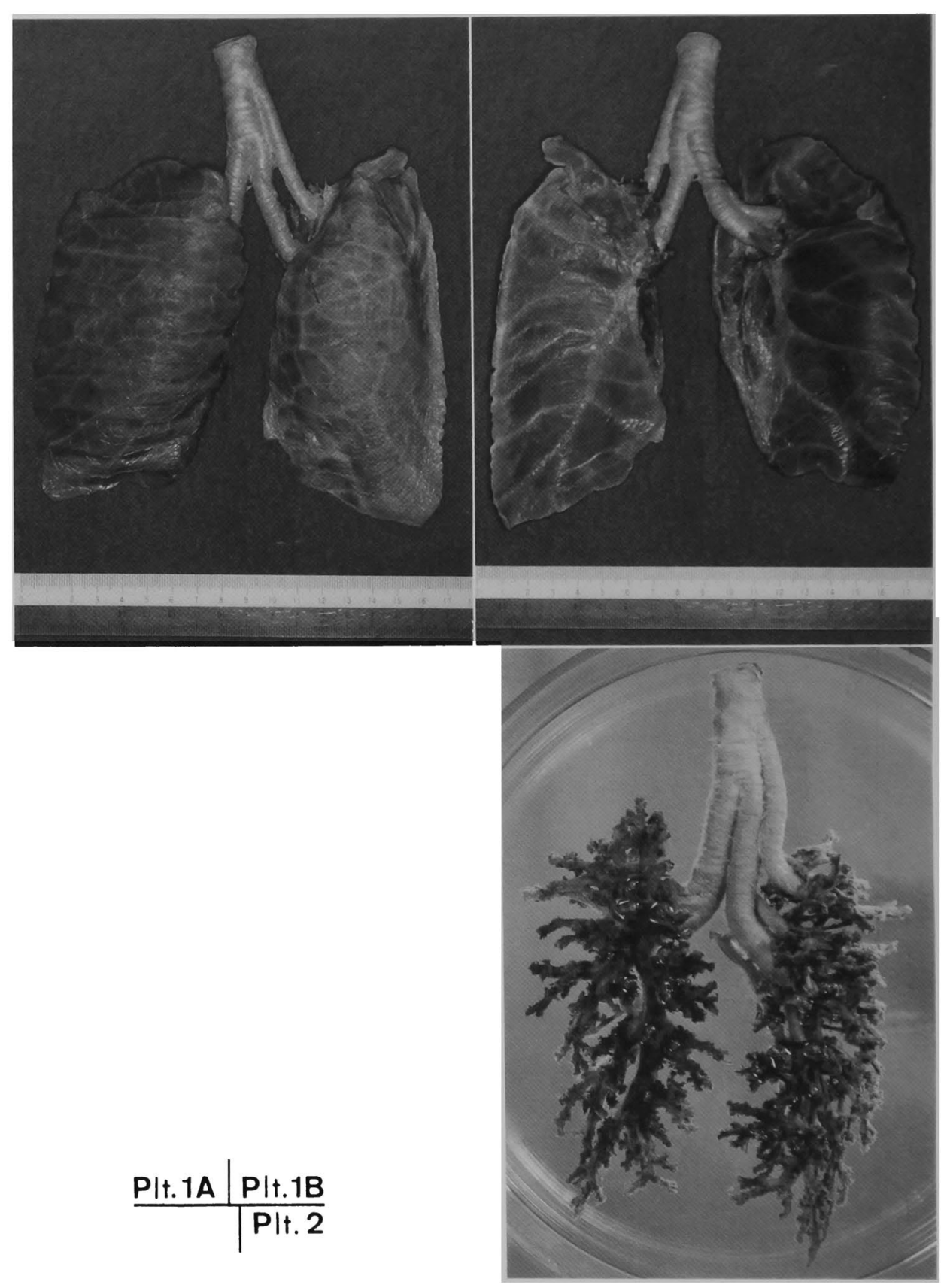

\section{Explanations of Figures}

Plate 1. Dorsal (A) and ventral (B) aspects of the lungs. The trachea separates into three. The first branch is ordinarily called the tracheal bronchus and is the common feature in cetacean lungs. Fine lobulation is observable through the visceral pleura. This lobulation is one of the conspicuous characteristics of the Ganges river dolphin.

Plate 2. Dorsal aspect of the tracheobronchial tree with pulmonary vessels. Bronchioles were almost entirely removed to observe the trunk and main branches. 